\title{
EDAD ÓSEA ESTIMADA POR LOS MÉTODOS TW2 Y TW3 EN MENORES INDÍGENAS DE OAXACA, MÉXICO
}

\section{BONE AGE ESTIMATION BY TW2 AND TW3 METHODS IN INDIGENOUS CHILDREN FROM OAXACA, MEXICO}

\author{
María Elena Sáenz Faulhaber*, Rosa María Ramos Rodríguez¹ y Andrés del Ángel Escalona \\ ${ }^{I}$ Instituto de Investigaciones Antropológicas. Universidad Nacional Autónoma de México. Ciudad de México. México
}

\section{PALABRAS CLAVE: maduración ósea; métodos TW2, TW3; población indígena; México}

\begin{abstract}
RESUMEN Por su vinculación con la edad cronológica y el estatus de maduración, la edad ósea es útil en los campos clínico, forense, legal y deportivo. En 1983 Tanner y colaboradores propusieron el método TW2, años más tarde lo modificaron denominándolo TW3. En Latinoamérica, los estudios en población abierta en los que se han estimado las edades óseas empleado dichos métodos son muy escasos y no se llegan a conclusiones precisas sobre la conveniencia de utilizar uno u otro, y en México no se cuenta con trabajo alguno publicado en el que se utilice el método TW3. Objetivo: explorar cual método (TW2 o el TW3) es más apropiado para estimar la edad ósea en menores que comparten ancestrías de origen indoamericano y que participan
\end{abstract}

de rasgos culturales similares. Se utilizaron radiografías de muñeca y mano obtenidas entre los años 1986 y 1987, en 393 menores (182 mujeres y 211 hombres) de 6 a 17 años de edad, de origen mixteco, residentes en la Heroica Ciudad de Tlaxiaco, Oaxaca, México. Para la estimación de la edad ósea se siguieron las técnicas RUS de TW2 y TW3. Se encontraron diferencias estadísticamente significativas entre la estimación de la edad ósea por los métodos TW2 y TW3. Entre las edades estimadas por el método TW2 y la edad cronológica se encontraron mayores diferencias que empleando el TW3, estimaciones derivadas que más se apegaron a la edad cronológica. Rev Arg Antrop Biol 22(1), 2020. doi: $10.24215 / 18536387 \mathrm{e} 015$

KEYWORDS bone maturation; methods TW2, TW3; indigenous population, Mexico

$A B S T R A C T$ In clinical, forensic, legal and sport fields it is usefull to estimate the chronological age as well as the maturation status and therefore it is useful to determine it through the bone age. In 1983 Tanner et al. proposed the TW2 method, which was later modified and renamed as TW3. In Latin America, bone age studies of open populations using the TW2 and TW3 methods were carried out, but none of them concludes which of both is more accurate and for Mexico there are no published data on TW3. Objective: to explore which method (TW2 or TW3) is most appropriate to estimate bone age of children who share indoamerican ancestry and participate of similar cultural traits. Based on X-Rays of hand and wrist obtained between 1986 and 1987 in 393 youngsters (182 girls and 211 boys) from 6 to 17 years of age of Mixtec origin from the Heroica Ciudad de Tlaxiaco, Oaxaca, Mexico, bone age was estimated folowing RUS TW2 and RUS TW3 methods. Significant statistical differences were found between TW2 and TW3. Greater differences were found using TW2 method vs chronological age, than using the TW3, derived estimates which lay closer to chronological age. Rev Arg Antrop Biol 22(1), 2020. doi:10.24215/18536387e015
Principalmente en los campos clínico, forense, legal y deportivo existe la necesidad de estimar la edad cronológica (EC) así como el estatus de maduración, de manera tal que la evaluación de la edad ósea (EO) resulta de gran utilidad para ello (Beh y Payne-James, 2011; Malina, 2011; Schmeling, Reisinger, Geserick y Olze, 2005; Schmidt, Nitz, Schulz y Schmeling, 2008). Desde el descubrimiento de los rayos $\mathrm{X}$, dada la accesibilidad a la región anatómica, los huesos de la muñeca y mano son los más utilizados para determinar el estatus de maduración ósea. Si bien los procesos que ocurren para alcanzar el estadio adulto en el desarrollo óseo son independientes de la edad cronológica (Cameron, 2015), ellos tienen lugar alrededor de un tiempo calendárico desde la vida intrauterina hasta que ocurre el cierre epifisiario, aun teniendo en consideración los ritmos de maduración pro-

Financiamiento: Instituto de Investigaciones Antropológicas de la Universidad Nacional Autónoma de México; Centre d'Hémotypologie del Centre National de la Recherche Scientifique de Francia.

*Correspondencia a: Rosa María Ramos Rodríguez. Instituto de Investigaciones Antropológicas. Universidad Nacional Autónoma de México. Ciudad Universitaria. Ciudad de México.04510 México. E-mail: rmrr2525@gmail.com

Recibido 2 Octubre 2018; aceptado 9 Julio 2019

doi: $10.24215 / 18536387 \mathrm{e} 015$ 
pios de cada persona (Tanner, 1978).

Todd (1937) elaboró el primer Atlas de maduración esquelética (Acheson, 1966), mismo que constituyó la base para formular el método propuesto por Greulich y Pyle (1959). En 1962 Tanner, Whitehouse y Healy, basados en las radiografías de mano y muñeca izquierdas, obtenidas en niños y jóvenes británicos, propusieron un método innovador (TW1) para estimar la EO de menores en crecimiento (Tanner, Whitehouse, Healy, 1962). En 1975 realizaron modificaciones a su técnica nombrándola como el método TW2, en el cual se podía elegir entre varios sistemas: a) el estudio de 20 huesos de muñeca, carpo, así como los metacarpianos y falanges I, III y V (técnica TW2-20), b) el estudio de los huesos cúbito, radio y carpales (técnica CAR) y c) el estudio de los huesos radio, cúbito y metacarpianos y falanges I, III y V (técnica RUS) (Tanner, Whitehouse, Marshall, Healy y Goldstein, 1975). Años después, al utilizar estos métodos en otras poblaciones no británicas (Kimura, 1977; Lejarraga, Guimarey y Orazi, 1997; Ye, Wang y Cao, 1992), se observó que la estimación de la maduración ósea reportaba en lo general valores más altos que los esperados para la correspondiente EC. Dado lo anterior, el grupo de investigadores británicos decidió incorporar datos de otras poblaciones del occidente de Europa, Estados Unidos de Norteamérica, Argentina y Japón, y eliminaron el sistema de 20 huesos porque duplicaba información sobre la maduración de los huesos de la mano, manteniendo únicamente los sistemas RUS y CAR. Además de lo anterior, sin modificar las estimaciones para los estadios de cada uno de los huesos seleccionados, se cambiaron únicamente las asignaciones correspondientes a cada EO. Con estas cifras propusieron el método TW3 (Tanner et al., 2001), lo que ha motivado la necesidad de explorar la conveniencia de utilizar uno u otro método (Büken et al., 2010; Haiter-Neto, Kurita, Menezes y Casanova, 2006; Kim, Lee y Yu, 2015; Ortega et al., 2006; Pinchi et al., 2014; Schmidt et al., 2008).

Sin que medie patología alguna o selección por actividad deportiva, en Latinoamérica son pocos los trabajos publicados en grupos de población abierta, en los que se ha estimado la edad ósea a través radiografías de muñeca y mano con los métodos TW2 y TW3; y debe señalarse que la mayoría de los grupos humanos estudiados tienen componente genético y cultural muy diverso (Jiménez, Berdasco, Ruben y Jordán, 1987; Landaeta y Lanes, 1991; Lejarraga et al., 1997; López, Morón y Urdaneta, 2008; López-Blanco, Macías, Izaguirre, Ortega et al., 2006). Por su parte, en México los estudios en población abierta en los que se han empleado alguno de los métodos mencionados también son escasos, y no se cuenta con publicación alguna en el que se utilice la técnica TW3 (por ejemplo Faulhaber, 1981; Malina y Little, 1981; Malina, Himes y Stepick, 1976; Peña y Cárdenas, 1996; Sáenz Faulhaber, 1980, 1981, 1989; Villanueva, Sáenz y Serrano, 1984).

Por lo anterior, debido a la escasa información sobre el comportamiento de las estimaciones de la edad esquelética según los métodos TW2 y TW3, resulta de interés corroborar si este último es más apropiado para la estimación de la edad cronológica en menores que comparten orígenes autóctonos indoamericanos y prácticas culturales similares.

\section{MATERIAL Y MÉTODOS}

En el año de 1985 se inició el proyecto de investigación "Biología humana y desarrollo en la Mixteca Alta", en el estado de Oaxaca, México, apoyado por el Instituto de Investigaciones Antropológicas de la Universidad Nacional Autónoma de México (UNAM) y el Centre d'Hémotypologie del Centre National de la Recherche Scientifique de Francia. Como parte de dicho proyecto, durante los meses de abril de 1986 y febrero de 1987, además de recabar información antropométrica (Ramos Rodríguez, 2004, 2005), se obtuvieron radiografías de muñeca y mano de 393 menores de entre 6 y 17 años de edad (182 mujeres y 211 hombres) residentes en la Heroica Ciudad de Tlaxiaco, los cuales asistían a dos escuelas públicas, una primaria y otra secundaria (en los turnos matutino y vespertino). Esta ciudad se localiza en el municipio del mismo nombre en el estado de Oaxaca, México (Fig. 1) ubicada 


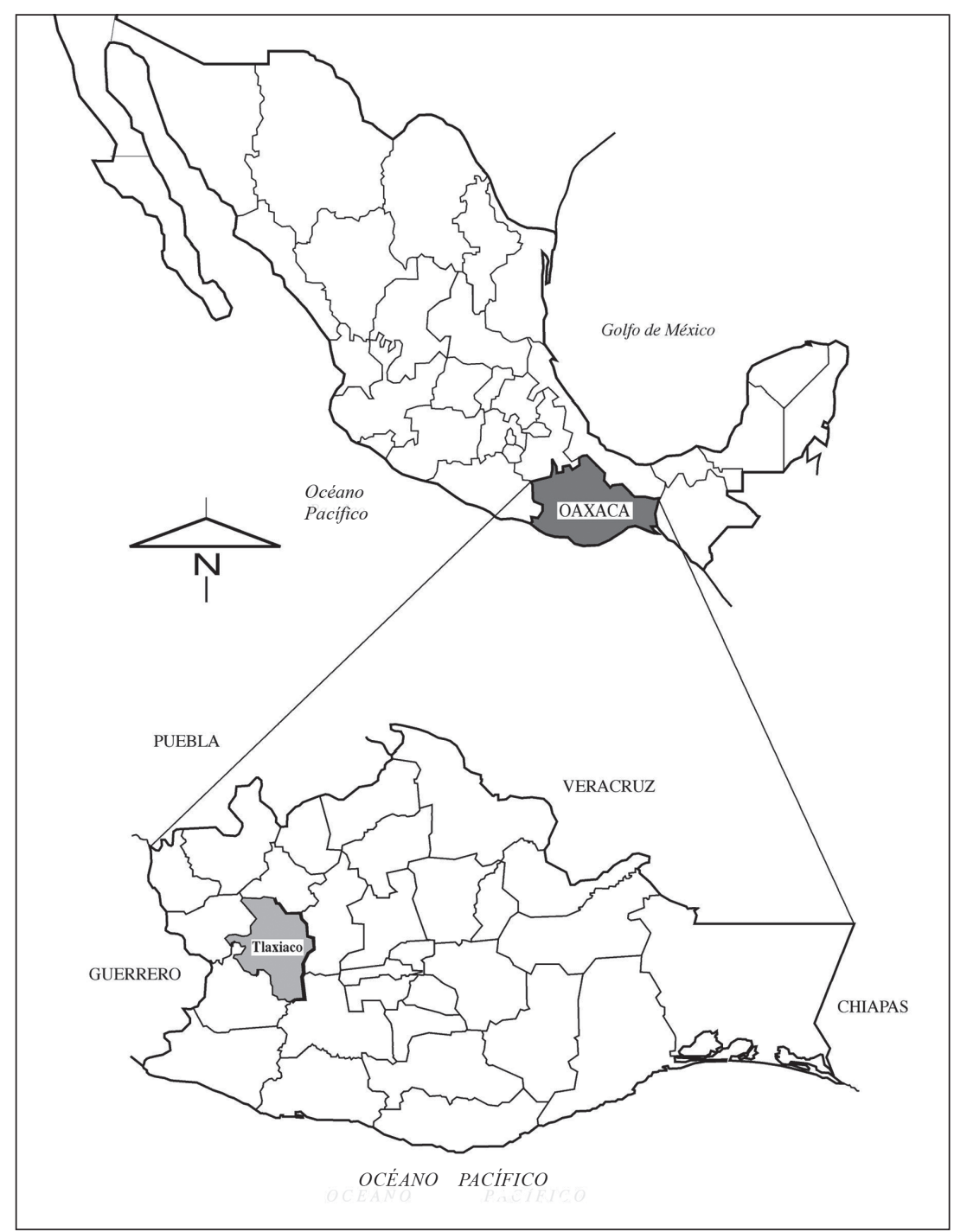

Fig. 1. Localización del municipio de Tlaxiaco, Oaxaca, México.

en la región conocida como Mixteca Alta, en la cual se asienta, desde tiempos anteriores a la conquista española, población del grupo lingüístico mixteco.

Previo a la toma de datos, y contando con la aprobación del cuerpo directivo del Instituto de Investigaciones Antropológicas de la UNAM, se informó a las autoridades escolares, a los padres de familia y a los menores el trabajo que se deseaba realizar. Una vez que se contó con las autorizaciones respectivas, se prosiguió con la investigación.

La edad cronológica de cada menor se ob- tuvo de la documentación del plantel educativo donde se realizó la presente investigación. Es pertinente mencionar que dicha información se recabó a partir de las actas de nacimiento que cada escolar tenía que presentar para quedar inscrito en el centro educativo correspondiente. La toma de la radiografía de muñeca y mano se realizó de acuerdo con las recomendaciones de Tanner et al. (1975). La lectura de las radiografías se ejecutó por un solo observador (M.E.S.F.) y se definió la maduración ósea por los métodos TW2 y TW3, empleando el sistema RUS. En cada 
individuo de la muestra, y de acuerdo con el sexo, inicialmente se estimaron los valores para cada uno de los estadios de los huesos considerados. Con la suma parcial de ellos se obtuvo la puntuación total, misma que se contrastó con los referentes que aporta el sistema RUS, tanto para el método TW2 como para el TW3, y se obtuvo así la edad ósea de cada individuo.

En cada menor se calculó su edad decimal y los grupos de edad anuales se conformaron por intervalos definidos por $\pm 0,5$ años alrededor del entero (por ejemplo, el grupo de seis años incluyó a todos los menores cuya edad decimal se encontraba entre 5,50 y 6,49 años, y así sucesivamente).

Con el objetivo de evaluar la adecuación de los dos métodos de estimación de la EO (TW2 y TW3) a la EC, inicialmente se revisó la bibliografía sobre el tema. Los procedimientos analíticos puestos en práctica por los diversos autores iban del análisis de correlaciones hasta el de regresión, pasando por las pruebas pareadas y para muestras independientes. Así, ya que cada sujeto contaba con dos estimaciones por los métodos mencionados, siguiendo a Ortega et al. (2006), se tomó la decisión de utilizar la prueba de Dunnett (Fallas, 2012; Montgomery, 1991), en la que la EC (tratamiento de control) se comparó con las estimadas según TW2 y TW3 (tratamientos experimentales). A continuación, para abundar en el conocimiento de las diferencias entre los dos métodos de estimación de la edad ósea, debido al limitado número de casos por grupo de edad, se optó por evaluar las diferencias a través de la prueba no paramétrica de Wilcoxon para rangos con signo de pares comparados. Para resumir el comportamiento según el sexo de las distribuciones de las diferencias entre cada una de las estimaciones de las edades óseas y cronológica $(\mathrm{D}=\mathrm{EO}-\mathrm{EC})$ se empleó la representación de diagramas de caja. Para los cálculos se utilizó el paquete estadístico SPSS versión 23 para el sistema operativo Windows 10.

\section{RESULTADOS}

En la Tabla 1 se presentan por sexo y por grupo de edad el tamaño de muestra, la media y la desviación estándar de la edad cronológica (EC) y las edades óseas (EO) estimadas por los métodos TW2 y TW3 (sistema RUS).

La comparación en cada menor entre su EC y sus edades óseas muestran un comportamiento diferente entre hombres y mujeres. En los primeros, según el método TW2, entre los 7 y 12 años y a los 17 años no se encontraron diferencias estadísticamente significativas, mientras que a los 6 años y entre $\operatorname{los} 13$ y 16 años las diferencias fueron positivamente mayores. Por su parte, empleando el TW3, en general las estimaciones permanecieron similares en todos los grupos de edad, excepto a los 6, 7 y 12 años (Tabla 2).

En las mujeres únicamente en los grupos de 6 a 8 años no se observaron diferencias entre la EC y las EO (TW2 y TW3), sin embargo las diferencias de las EO estimadas por el TW2 fueron significativamente superiores entre los $9 \mathrm{y}$ 15 años. Debe destacarse que en todos los grupos de edad la EO por TW3 fue similar a la EC, salvo en el grupo de 16 años que fue significativamente mayor. El proceder descrito se ilustra en los diagramas de caja elaborados por sexo y grupo de edad (Tabla 3, Fig. 2).

En la Tabla 4 y la Figura 3 se ilustran las diferencias entre las estimaciones de la EO según los dos métodos ahora considerados (TW2 y TW3). Entre los hombres, desde los 6 a los 8 años las diferencias se inician con signo negativo, esto es que la edad estimada por el sistema TW2 es menor que la obtenida por el TW3, pero a partir de los diez años esta relación se invierte, pues se presentan con signo positivo y tienden a aumentar consistentemente hasta alcanzar el estadio adulto. En las mujeres se aprecia claramente la sobreestimación de la EO por TW2, entre los grupos de edad de 6 a 13 años, siempre mayor en ellas en relación con los hombres; entre los nueve años y hasta que ocurre el cierre de las epífisis, las diferencias se mantienen prácticamente iguales. Tanner et al. (2001) reportan que entre los sistemas RUS TW2 y RUS TW3 el patrón de las diferencias fue de un año entre los 5 y 14 años para las mujeres, y de los 5 a los 16 años para los hombres; sin embargo, en el presente trabajo esto sucede a partir de los ocho años en las mujeres (más cercano a lo reportado en el estudio británi- 
TABLA 1. Estadísticos descriptivos de las edades cronológica y ósea estimadas por los sistemas RUS TW2 y TW3, según sexo y grupo de edad cronológica

\begin{tabular}{|c|c|c|c|c|c|c|c|c|}
\hline \multirow[b]{2}{*}{ Sexo } & \multirow[b]{2}{*}{$\begin{array}{c}\text { Grupo } \\
\text { de edad }\end{array}$} & \multirow[b]{2}{*}{$n$} & \multicolumn{2}{|c|}{ Edad cronológica } & \multicolumn{2}{|c|}{ TW2 } & \multicolumn{2}{|c|}{ TW3 } \\
\hline & & & Media & DE & Media & DE & Media & DE \\
\hline \multirow[t]{12}{*}{ Hombres } & 6 & 14 & 6,26 & 0,12 & 6,99 & 0,58 & 7,18 & 0,57 \\
\hline & 7 & 19 & 6,93 & 0,35 & 7,33 & 0,76 & 7,51 & 0,71 \\
\hline & 8 & 19 & 8,01 & 0,28 & 8,08 & 1,10 & 8,18 & 0,96 \\
\hline & 9 & 17 & 8,83 & 0,34 & 8,49 & 1,07 & 8,54 & 0,88 \\
\hline & 10 & 20 & 9,97 & 0,31 & 10,33 & 1,25 & 9,95 & 0,87 \\
\hline & 11 & 10 & 10,86 & 0,26 & 11,29 & 1,01 & 10,56 & 0,61 \\
\hline & 12 & 31 & 12,02 & 0,22 & 12,07 & 1,29 & 11,22 & 0,94 \\
\hline & 13 & 22 & 13,03 & 0,20 & 13,77 & 1,23 & 12,72 & 1,23 \\
\hline & 14 & 14 & 14,02 & 0,36 & 14,84 & 1,37 & 13,80 & 1,50 \\
\hline & 15 & 22 & 15,01 & 0,30 & 16,31 & 1,23 & 15,27 & 1,03 \\
\hline & 16 & 13 & 15,91 & 0,23 & 17,33 & 1,08 & 16,05 & 0,80 \\
\hline & 17 & 10 & 16,75 & 0,21 & 17,44 & 1,08 & 16,07 & 0,71 \\
\hline \multirow[t]{11}{*}{ Mujeres } & 6 & 6 & 6,29 & 0,17 & 6,27 & 1,28 & 5,72 & 0,97 \\
\hline & 7 & 14 & 7,10 & 0,30 & 7,11 & 1,16 & 6,39 & 0,93 \\
\hline & 8 & 21 & 8,02 & 0,34 & 8,53 & 1,39 & 7,62 & 1,26 \\
\hline & 9 & 14 & 8,96 & 0,26 & 10,04 & 1,00 & 8,97 & 0,98 \\
\hline & 10 & 18 & 10,09 & 0,22 & 11,01 & 1,07 & 9,94 & 1,05 \\
\hline & 11 & 12 & 11,08 & 0,31 & 12,35 & 1,27 & 11,27 & 1,29 \\
\hline & 12 & 22 & 11,92 & 0,24 & 13,06 & 0,82 & 12,00 & 0,87 \\
\hline & 13 & 14 & 13,00 & 0,24 & 13,82 & 1,13 & 12,76 & 1,21 \\
\hline & 14 & 20 & 14,08 & 0,33 & 15,32 & 0,89 & 14,45 & 0,73 \\
\hline & 15 & 21 & 14,92 & 0,31 & 15,85 & 0,32 & 14,90 & 0,20 \\
\hline & 16 & 20 & 15,92 & 0,27 & 15,88 & 0,30 & 14,90 & 0,28 \\
\hline
\end{tabular}

$\mathrm{DE}=$ desviación estándar.

co), mientras que en los hombres estas diferencias fueron mínimas entre los 6 y los 11 años $y$, a partir de entonces, se situaron en alrededor de un año.

En la figura 4, en la que se agrupan las diferencias, según el sexo, sin considerar grupo de edad, se constata que, en contraste con las edades estimadas por TW2, el método TW3 proporciona estimaciones muy cercanas a la edad cronológica de los sujetos, sobre todo en el caso de los hombres, en los que no se encontraron diferencias con significado estadístico, mientras que en las mujeres la diferencia sí lo fue (Tabla 5). Si se consideran los valores de $t$ de las pruebas, se destaca que la magnitud de las diferencias son varias veces mayores empleando el método TW2. Además, se observó mayor precisión en las estimaciones derivadas del método TW3, debido a la menor amplitud de las cajas: hombres, 1,17 DE vs. 1,01 DE y en mujeres de 1,02 DE vs. $0,95 \mathrm{DE}$. 
TABLA 2. Significado de las diferencias individuales entre la edad cronológica (EC) y las edades óseas (EO) estimadas por los métodos RUS TW2 y RUS TW3, en años. Prueba $\mathrm{t}$ de Dunnett bilateral. Hombres

\begin{tabular}{|c|c|c|c|c|}
\hline Edad & Diferencias entre EO y EC según método & Media & Error estándar & $p$ \\
\hline \multirow{2}{*}{6} & TW2-EC & 0,724 & 0,179 & $<0,001$ \\
\hline & TW3-EC & 0,916 & 0,179 & $<0,001$ \\
\hline \multirow{2}{*}{7} & TW2-EC & 0,397 & 0,205 & 0,105 \\
\hline & TW3-EC & 0,576 & 0,205 & 0,013 \\
\hline \multirow{2}{*}{8} & TW2-EC & 0,071 & 0,278 & 0,954 \\
\hline & TW3-EC & 0,166 & 0,278 & 0,777 \\
\hline \multirow{2}{*}{9} & TW2-EC & $-0,342$ & 0,283 & 0,383 \\
\hline & TW3-EC & $-0,295$ & 0,283 & 0,482 \\
\hline \multirow{2}{*}{10} & TW2-EC & 0,350 & 0,283 & 0,366 \\
\hline & TW3-EC & $-0,026$ & 0,283 & 0,994 \\
\hline \multirow{2}{*}{11} & TW2-EC & 0,428 & 0,311 & 0,301 \\
\hline & TW3-EC & $-0,302$ & 0,311 & 0,529 \\
\hline \multirow{2}{*}{12} & TW2-EC & 0,050 & 0,236 & 0,968 \\
\hline & TW3-EC & $-0,802$ & 0,236 & 0,002 \\
\hline \multirow{2}{*}{13} & TW2-EC & 0,746 & 0,306 & 0,033 \\
\hline & TW3-EC & $-0,309$ & 0,306 & 0,499 \\
\hline \multirow{2}{*}{14} & TW2-EC & 0,826 & 0,451 & 0,132 \\
\hline & TW3-EC & $-0,217$ & 0,451 & 0,846 \\
\hline \multirow{2}{*}{15} & TW2-EC & 1,303 & 0,284 & $<0,001$ \\
\hline & TW3-EC & 0,262 & 0,284 & 0,557 \\
\hline \multirow{2}{*}{16} & TW2-EC & 1,418 & 0,308 & $<0,001$ \\
\hline & TW3-EC & 0,133 & 0,308 & 0,874 \\
\hline \multirow{2}{*}{17} & TW2-EC & 0,693 & 0,338 & 0,090 \\
\hline & TW3-EC & $-0,677$ & 0,338 & 0,099 \\
\hline
\end{tabular}

\section{DISCUSIÓN}

Sin duda continúa la necesidad, especialmente en el campo médico legal y forense, de definir cuál es mejor método o métodos basados en la maduración esquelética, que den mayor certeza en la estimación de la EC. Los métodos apoyados en la maduración ósea de muñeca y mano que más se han utilizado son el Greulich y Pyle (1959) y los propuestos por Tanner y colaboradores, ya sea el TW1, que dieron paso al TW2 y posteriormente, para mejorar su aplicabilidad, al TW3 (Tanner et al., 1962, 1975, 2001).

Diversos trabajos se han enfocado a compa- rar los resultados de la maduración y estimación de la edad cronológica, especialmente aplicando los métodos de Greulich-Pyle, TW2 y TW3 y otros referentes propuestos (Cole, et al. 2005; Ontell, Ivanovic, Ablin y Barlow, 1996; Schmeling et al., 2000; Tanner et al., 2001). Algunos más tratan de corroborar específicamente la aplicabilidad del método TW3 para observar la maduración esquelética (Bertaina, Stasiowska, Benso y Vannelli, 2007; López et al., 2008). Los resultados obtenidos varían y no se llega a conclusiones mayoritarias que den mayor certeza aplicando uno u otro método. De hecho, tratándose de un tema tan delicado cuando se implican asuntos legales o forenses se sugiere 
TABLA 3. Significado de las diferencias individuales entre la edad cronológica (EC) y las edades óseas (EO) estimadas por los métodos RUS TW2 y RUS TW3, en años. Prueba $\mathrm{t}$ de Dunnett bilateral. Mujeres

\begin{tabular}{|c|c|c|c|c|}
\hline Edad & Diferencias entre EO y EC según método & Media & Error estándar & $p$ \\
\hline \multirow{2}{*}{6} & TW2-EC & $-0,027$ & 0,539 & 0,998 \\
\hline & TW3-EC & $-0,577$ & 0,539 & 0,475 \\
\hline \multirow{2}{*}{7} & TW2-EC & 0,017 & 0,331 & 0,998 \\
\hline & TW3-EC & $-0,704$ & 0,331 & 0,072 \\
\hline \multirow{2}{*}{8} & TW2-EC & 0,507 & 0,339 & 0,240 \\
\hline & TW3-EC & $-0,403$ & 0,339 & 0,392 \\
\hline \multirow{2}{*}{9} & TW2-EC & 1,074 & 0,310 & 0,003 \\
\hline & TW3-EC & 0,009 & 0,310 & 0,999 \\
\hline \multirow{2}{*}{10} & TW2-EC & 0,926 & 0,291 & 0,005 \\
\hline & TW3-EC & $-0,141$ & 0,291 & 0,846 \\
\hline \multirow{2}{*}{11} & TW2-EC & 1,275 & 0,433 & 0,011 \\
\hline & TW3-EC & 0,192 & 0,433 & 0,868 \\
\hline \multirow{2}{*}{12} & TW2-EC & 1,135 & 0,212 & 0,000 \\
\hline & TW3-EC & 0,076 & 0,212 & 0,910 \\
\hline \multirow{2}{*}{13} & TW2-EC & 0,819 & 0,365 & 0,056 \\
\hline & TW3-EC & $-0,245$ & 0,365 & 0,727 \\
\hline \multirow{2}{*}{14} & TW2-EC & 1,238 & 0,218 & 0,000 \\
\hline & TW3-EC & 0,373 & 0,218 & 0,163 \\
\hline \multirow{2}{*}{15} & TW2-EC & 0,934 & 0,087 & 0,000 \\
\hline & TW3-EC & $-0,013$ & 0,087 & 0,983 \\
\hline \multirow{2}{*}{16} & TW2-EC & $-0,040$ & 0,090 & 0,870 \\
\hline & TW3-EC & $-1,025$ & 0,090 & 0,000 \\
\hline
\end{tabular}

tener cautela con las estimaciones de la EC, e incluso se expresa la conveniencia de combinar diversos métodos (Schmeling et al., 2005). En general, los estudios dedicados a comparar las estimaciones de EO según métodos TW2 y TW3, observan mayores diferencias entre la EC y la EO por el método TW2 y mucho más asociación entre la EC y la EO por el método TW3.

En el campo de la Biología Humana y la Antropología Física o Biológica es importante contar con explicaciones que den cuenta de la variabilidad humana y puedan generarse y validarse materiales útiles para aplicarse en otros campos de conocimiento, de ahí el interés de evaluar la adecuación de los dos métodos de estimación de la EO (TW2 y TW3) a la EC en población con alta ancestría de origen indoamericano y que participan de rasgos culturales comunes.
Es innegable que la maduración, como en tantos otros procesos biológicos, el componente genético, las mediaciones epigenéticas y las interacciones que ocurren entre el sujeto y su entorno son determinantes en la expresión de los propios procesos de maduración (Little y Malina, 2007). Así, diversos trabajos que exploran la influencia de la etnicidad sobre la maduración ósea (Cole et al., 2015; López et al., 2008; Ontell et al., 1996; Schmeling et al., 2000) en general concluyen que las diferencias encontradas se deben principalmente al nivel socioeconómico, que puede impactar (favorable o desfavorablemente) en los procesos de crecimiento y desarrollo físico.

En relación con las diferencias entre las estimaciones de la EO por TW2 y TW3, y a pesar de que la población brasileña estudiada 


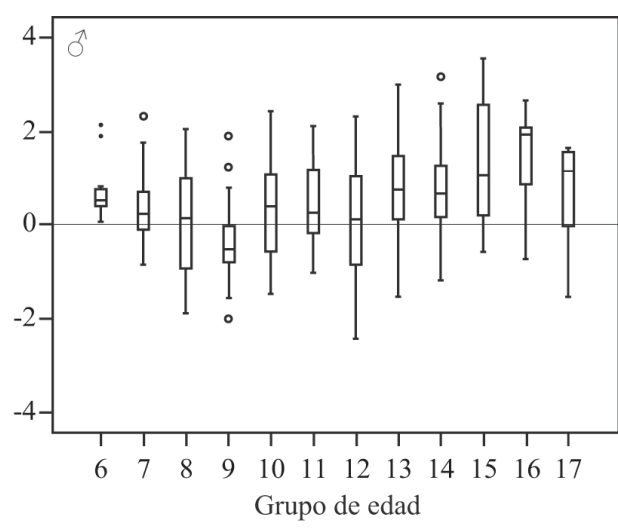

TW2

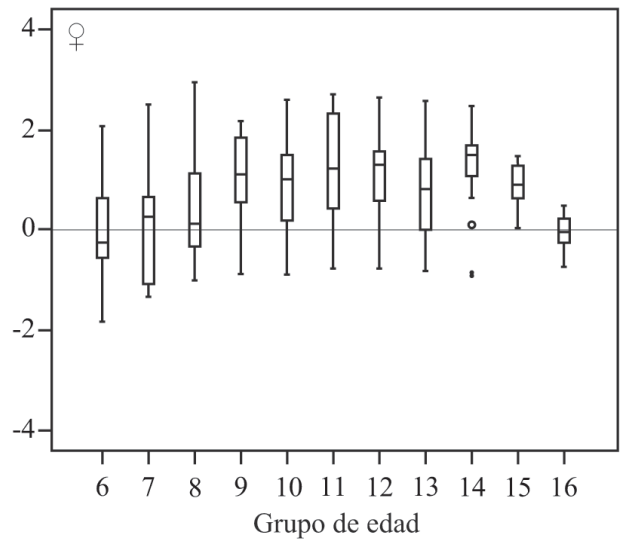

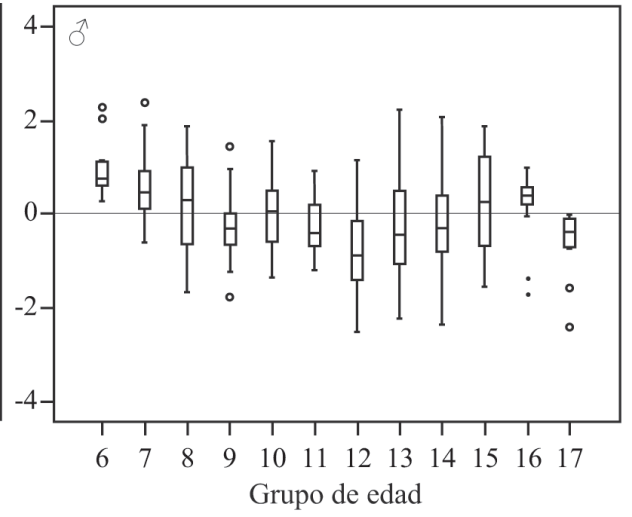

TW3

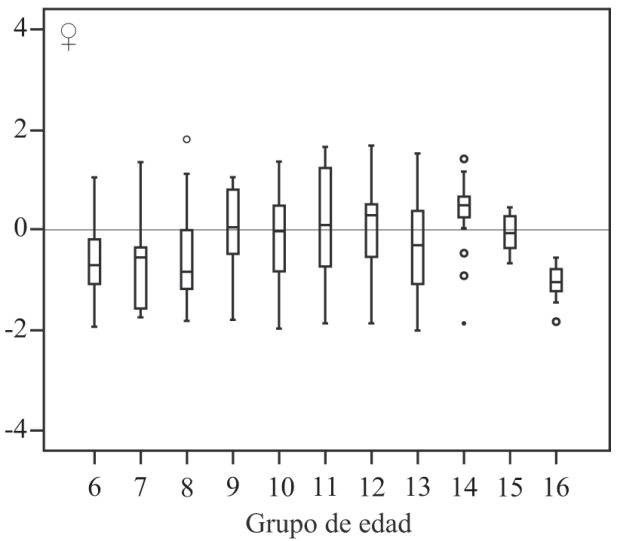

Fig. 2. Diagramas de caja de las diferencias entre edad ósea y la edad cronológica $(\mathrm{D}=\mathrm{EO}-\mathrm{EC})$ reportadas para hombres (hilera superior) y para mujeres (hilera inferior) según los dos métodos de estimación de edad ósea por grupos de edad y sexo.

por Ortega et al. (2006) tiene características biológicas y culturales diferentes a las del presente estudio, éste arrojó resultados similares. En efecto, en las mujeres, las diferencias fueron todas positivas desde las edades más tempranas estudiadas, en promedio de poco más de 10 meses, en contraste con los 11 meses en promedio reportado por el estudio en población brasileña. En los hombres las diferencias se inician con valores negativos que van de $-0,193$ a 0,852 en las edades más tempranas (6-12 años) y posteriormente varían alrededor de un año en las siguientes, mientras que en los brasileños suceden casi en la misma magnitud, pero entre $\operatorname{los} 7$ y 10 años.

En el trabajo que ahora se discute, se ha demostrado que para la población estudiada, esto es, menores residentes en la $\mathrm{H}$. Ciudad de Tlaxiaco, los cuales comparten ancestrías comunes y características culturales propias del grupo mixteco, la estimación de la EO por el método TW3 se encuentra más cercana a la EC, similares resultados a los reportados por Schmidt et al. (2008). A su vez, se observó un comportamiento diferente según sexo y grupo de edad, ya que en las niñas se apreció franca consistencia en la similitud entre la EO estimada por dicho método y la EC en todos los grupos de edad, mientras que en los niños no fue así. Sin embargo, cuando las diferencias en las estimaciones de la EO y EC se agruparon únicamente según el sexo, la tendencia general fue a encontrar mayor asociación entre ellas en el grupo masculino en su conjunto, no así en las mujeres, en quienes en promedio, la edad estimada por TW3 fue significativamente menor a la edad cronológica, similar a los hallazgos de Pinchi et al. (2014). 
TABLA 4. Comparación pareada de las diferencias de edades óseas según los sistemas TW2 y TW3, por grupo de edad y sexo. Prueba de Wilcoxon para rangos con signo de pares comparados

\begin{tabular}{|c|c|c|c|c|c|}
\hline Grupo de edad & $n$ & Media & DE & $z$ & $p$ \\
\hline \multicolumn{6}{|l|}{ Hombres } \\
\hline 6 & 14 & $-0,193$ & 0,083 & $-3,27$ & 0,001 \\
\hline 7 & 19 & $-0,179$ & 0,071 & $-3,86$ & $<0,001$ \\
\hline 8 & 19 & $-0,095$ & 0,154 & $-2,35$ & 0,019 \\
\hline 9 & 17 & $-0,047$ & 0,210 & $-1,31$ & 0,189 \\
\hline 10 & 20 & 0,375 & 0,385 & $-3,36$ & 0,001 \\
\hline 11 & 10 & 0,730 & 0,435 & $-2,81$ & 0,005 \\
\hline 12 & 31 & 0,852 & 0,362 & $-4,87$ & $<0,001$ \\
\hline 13 & 22 & 1,055 & 0,168 & $-4,14$ & $<0,001$ \\
\hline 14 & 14 & 1,043 & 0,174 & $-3,31$ & 0,001 \\
\hline 15 & 22 & 1,041 & 0,336 & $-4,13$ & $<0,001$ \\
\hline 16 & 13 & 1,285 & 0,410 & $-3,22$ & 0,001 \\
\hline 17 & 10 & 1,370 & 0,432 & $-2,87$ & 0,004 \\
\hline \multicolumn{6}{|l|}{ Mujeres } \\
\hline 6 & 6 & 0,550 & 0,315 & $-2,21$ & 0,027 \\
\hline 7 & 14 & 0,721 & 0,249 & $-3,32$ & 0,001 \\
\hline 8 & 21 & 0,910 & 0,264 & $-4,02$ & $<0,001$ \\
\hline 9 & 14 & 1,064 & 0,074 & $-3,38$ & 0,001 \\
\hline 10 & 18 & 1,067 & 0,069 & $-3,80$ & $<0,001$ \\
\hline 11 & 12 & 1,083 & 0,058 & $-3,17$ & 0,002 \\
\hline 12 & 22 & 1,059 & 0,073 & $-4,19$ & $<0,001$ \\
\hline 13 & 14 & 1,064 & 0,084 & $-3,34$ & 0,001 \\
\hline 14 & 20 & 0,865 & 0,560 & $-3,31$ & 0,001 \\
\hline 15 & 21 & 0,948 & 0,125 & $-4,29$ & $<0,001$ \\
\hline 16 & 20 & 0,985 & 0,067 & $-4,23$ & $<0,001$ \\
\hline
\end{tabular}

$\mathrm{DE}=$ desviación estándar.

TABLA 5. Significado de las diferencias entre edad ósea y edad cronológica por sexo. Prueba $\mathrm{t}$ de Student para una muestra (valor de prueba: 0)

\begin{tabular}{ccccc}
\hline Sexo & \multicolumn{2}{c}{ TW2 } & \multicolumn{2}{c}{ TW3 } \\
\hline & $t$ & $p$ & $t$ & $p$ \\
Hombres & 6,34 & $<0,001$ & $-1,05$ & 0,295 \\
Mujeres & 9,68 & $<0,001$ & $-3,30$ & 0,001 \\
\hline
\end{tabular}




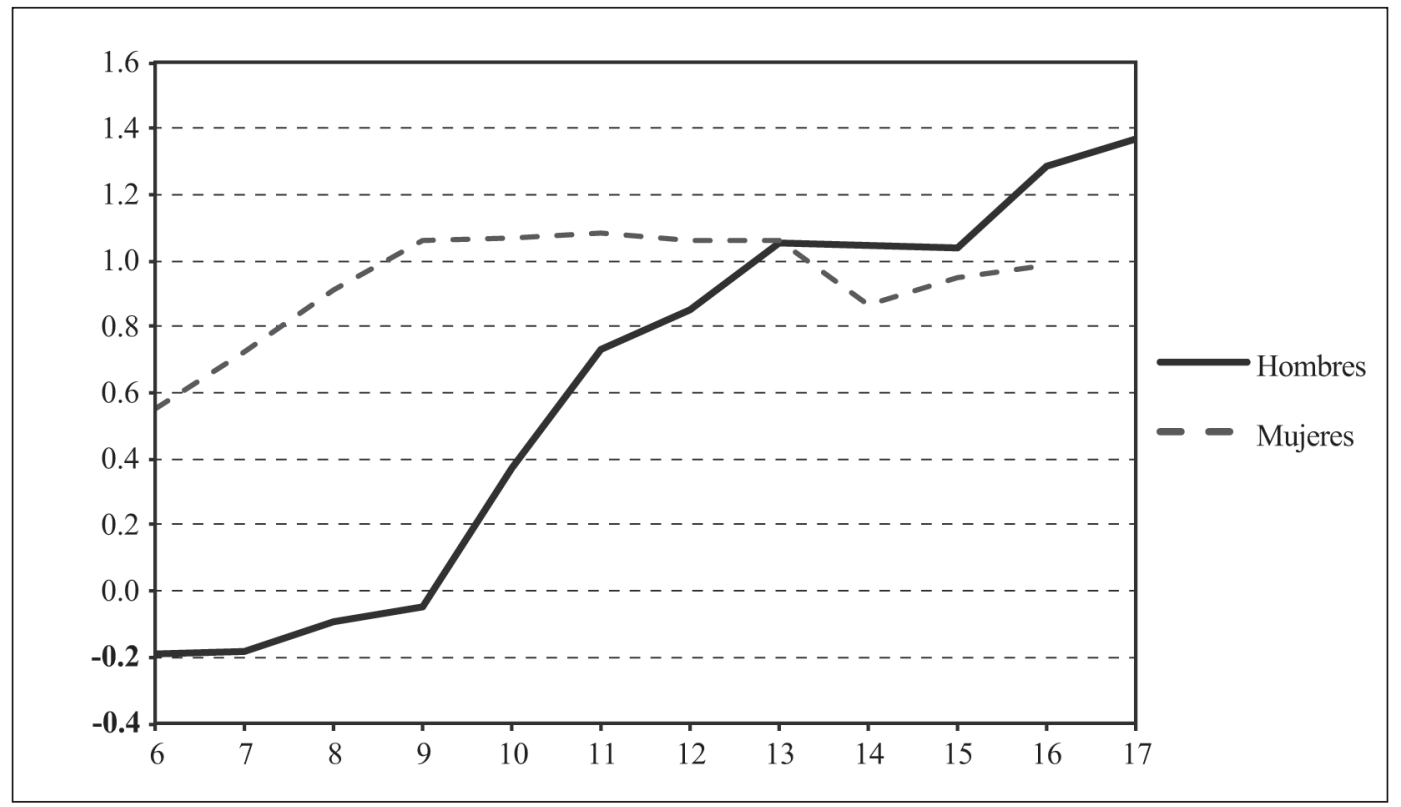

Fig. 3. Diferencias entre las estimaciones de la edad ósea según los sistemas TW2 y TW3 en hombres y mujeres.

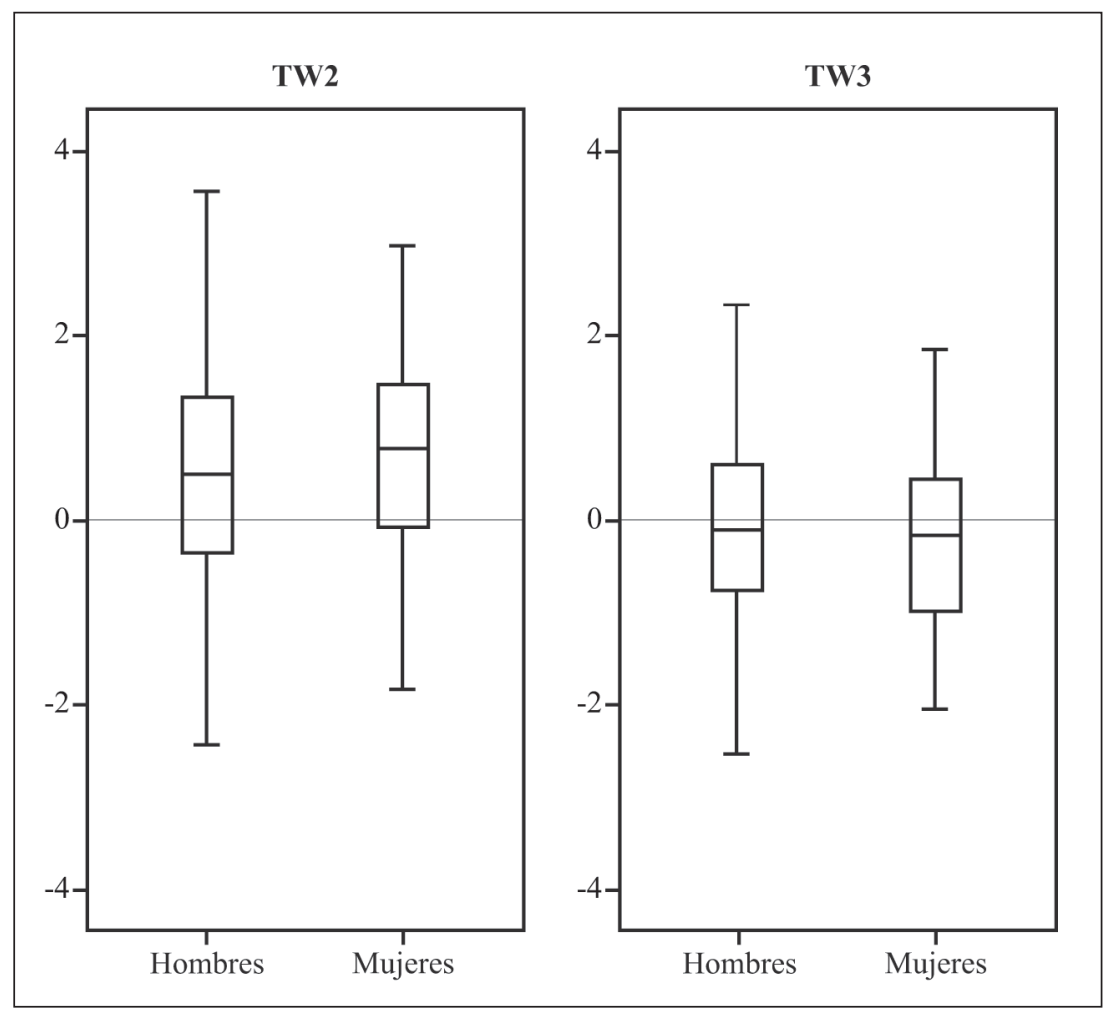

Fig. 4. Diagramas de caja de las diferencias entre edad ósea y edad cronológica $(\mathrm{D}=\mathrm{EO}-\mathrm{EC})$ según sexo. 


\section{CONCLUSIONES}

El objetivo de este estudio fue explorar cuál es el método de estimación de la edad ósea (TW2, TW3) que más se aproxima a la edad cronológica en menores que comparten orígenes autóctonos indoamericanos.

Los resultados del estudio nos llevan a plantear las siguientes conclusiones:

1-El método TW2 proporciona estimaciones significativamente mayores en ambos sexos y en la mayoría de los grupos de edad.

2-El TW3 proporciona estimaciones más adecuadas a la edad cronológica tanto en hombres como en mujeres.

3-Para conocer el comportamiento de las diferencias en las estimaciones de las EO según métodos TW2 y TW3, es conveniente realizar las estimaciones en la misma muestra de estudio.

4-Es necesario realizar más estudios en poblaciones con componente genético un tanto similar, y con similares condiciones materiales en las que ha transcurrido la vida de los menores estudiados para explicar la fluctuación de las diferencias entre las estimaciones de edad entre hombres y mujeres, según grupo etáreo.

\section{AGRADECIMIENTOS}

A los menores que participaron desinteresadamente en el trabajo de investigación, así como a sus padres y a las autoridades escolares que facilitaron el trabajo en las escuelas seleccionadas. A los doctores Luis Alberto Vargas y Carlos Serrano del Instituto de Investigaciones Antropológicas de la UNAM y Philippe Lefevre-Witier (†) del Centre d'Hémotypologie del Centre National de la Recherche Scientifique de Francia, quienes encabezaron el Proyecto Biología humana y desarrollo en la Mixteca Alta, Oaxaca. Al Instituto de Investigaciones Antropológicas de la Universidad Nacional Autónoma de México que financió el trabajo de campo y al Centre d'Hémotypologie del Centre National de la Recherche Scientifique de Francia que proporcionó las placas radiográficas.

\section{LITERATURA CITADA}

Acheson, R. M. (1966). Maturation of the skeleton. En F Falkner (Ed.), Human development (pp. 465-502). Filadelfia, EUA: WB Saunders.
Beh, P. y Payne-James, J. (2011). Clinical and legal requirements for age determination in the living. En S Black, A Aggrawal, J Payne-James (Eds.), Age estimation in the living: the practitioner's guide (pp. 30-42). Southern Gate, Chichester, West Sussex, Reino Unido: John Wiley \& Sons. doi:https://doi.org/10.1002/9780470669785. ch3

Bertaina, C., Stasiowska, B., Benso, A. y Vannelli, S. (2007). Is TW3 height prediction more accurate than TW2? Preliminary data. Hormone Research in Paediatrics, 67(5), 220-223. doi:doi.org/10.1159/000097439

Büken, B., Şafak, A. A., Büken, E., Yazici, B., Erkol, Z. y Erzengin, Ö. U. (2010). Is the Tanner-Whitehouse (TW3) method sufficiently reliable for forensic age determination of Turkish children? Turkish Journal of Medical Sciences, 40(5), 797-805. doi:10.3906/sag-0808-6

Cameron, N. (2015) Can maturity indicators be used to estimate chronological age in children? Annals of Human Biology, 42(4), 300-305. doi:10.3109/03014460.2015.1 032349

Cole, T. J., Rousham, E. K., Hawley, N. L., Cameron, N., Norris, S. A. y Pettifor, J. M. (2015). Ethnic and sex differences in skeletal maturation among the Birth to Twenty cohort in South Africa. Archives of Disease in Childhood, 100, 138-143. doi:http://dx.doi.org/10.1136/ archdischild-2014-306399

Fallas, J. (2012). Análisis de varianza. http://www.ucipfg. com/Repositorio/MGAP/MGAP-05/BLOQUE-ACADEMICO/Unidad-2/complementarias/analisis_de_varianza_2012.pdf. Consulta: 3 de mayo de 2018.

Faulhaber, J. (1981). La edad ósea de un grupo de niños mexicanos determinada según los métodos TW1 y TW2. Anales de Antropología, 18(1): 287-297. http:// revistas.unam.mx/index.php/antropologia/article/ view/23950/pdf 832

Greulich, W. W. y Pyle S. I. (1959). Radiographic atlas of skeletal development of the hand and wrist. Stanford, California, EUA: Stanford University Press.

Haiter-Neto, F., Kurita, L. M., Menezes, A. V. y Casanova, M. S. (2006). Skeletal age assessment: a comparison of 3 methods. American Journal of Orthodontics and Dentofacial Orthopedics, 130(4), 435-e15-435-e20. doi:https://doi.org/10.1016/j.ajodo.2006.03.023

Jiménez Hernández, J. M., Berdasco Gómez, A., Rubén Quesada, M. y Jordán, J. (1987). Estadios de maduración ósea por el método TW-2 y algunos datos sobre talla y menarquia en la población cubana. Revista Cubana de Pediatría, 59(6), 889-903. http://files.sld.cu/digitalizacion-bmn/files/2016/12/S0034-7531.1987.0006.0002. pdf

Kim, J. R., Lee, Y. S. y Yu, J. (2015). Assessment of bone age in prepubertal healthy Korean children: comparison among the Korean standard bone age chart, GreulichPyle method, and Tanner-Whitehouse method. Korean Journal of Radiology, 16(1): 201-205. https://doi. org/10.3348/kjr.2015.16.1.201

Kimura, K. (1977). Skeletal maturity of the hand and wrist in Japanese children by the TW2 method. Annals of Human Biology, 4(4): 353-356. doi:https://doi. org/10.1080/03014467700002281

Lejarraga, H., Guimarey, L. y Orazi, V. (1997). Skeletal maturity of the hand and wrist of healthy Argentinean children aged 4-12 years, assessed by the TWII method. Annals of Human Biology, 24(3), 257-261. doi:https:// doi.org/10.1080/03014469700004982

Little, B. B. y Malina, R. M. (2007). Gene-environment interaction in skeletal maturity and body dimensions of urban Oaxaca Mestizo schoolchildren. Annals 
of Human Biology, 34(2), 216-225. doi:https://doi. org $/ 10.1080 / 03014460601144011$

López, P., Morón, A. y Urdaneta, O. (2008). Maduración ósea de niños escolares (7-14 años) de las etnias Wayúu y Criolla del Municipio Maracaibo, Estado Zulia. Estudio Comparativo. Ciencia Odontológica, 5(2), 99-111. doi:http://produccioncientificaluz.org/index.php/cienciao/article/view/702

López-Blanco, M., Macías-Tomei, C., Izaguirre-Espinoza, I., Landaeta-Jiménez, M. y Lanes, R. (1991). Crecimiento, desarrollo, nutrición y adolescencia. En M López-Blanco y M Landaeta-Jiménez (Eds). Crecimiento y maduración: orientación diagnóstica. Manual de Crecimiento y Desarrollo (pp. 9-15). Caracas, Venezuela: Laboratorios Serono, FUNDACREDESA.

Malina, R. M., Himes, J. H. y Stepick, C. D. (1976). Skeletal maturity of the hand and wrist in Oaxaca school children. Annals of Human Biology, 3(3), 211-219. doi:https://doi.org/10.1080/03014467600001371

Malina, RM (2011). Skeletal age and age verification in youth sport. Sports Medicine, 41(11), 925-947. doi:https://doi. org/10.2165/11590300-000000000-00000

Malina, RM y Little, BB (1981). Comparison of TW1 and TW2 skeletal age differences in American Black and White and in Mexican children 6-13 years of age. Annals of Human Biology, 8(6), 543-548. doi:https://doi. org/10.1080/03014468100005381

Montgomery, DC (1991). Diseño y análisis de experimentos. México, Grupo Editorial Iberoamérica.

Ontell, F. K., Ivanovic, M., Ablin, D. S. y Barlow, T. W. (1996). Bone age in children of diverse ethnicity. American Journal of Roentgenology, 167(6), 1395-1398. doi:https://doi.org/10.2214/ajr.167.6.8956565

Ortega, A. I., Haiter-Neto, F., Ambrosano, G. M. B., Bóscolo, F. N., Almeida, S. M. y Casanova, M. S. (2006). Comparison of TW2 and TW3 skeletal age differences in a Brazilian population. Journal of Applied Oral Science, 14(2), 142-146. doi:http://dx.doi.org/10.1590/ S1678-77572006000200014

Peña, M. E. y Cárdenas, E. (1996). Los estudios de crecimiento y maduración esquelética. Su aplicación al campo de la salud. En S López Alonso, C Serrano Sánchez, L Márquez Morfín (Eds.). La antropología fisica en México. Estudios sobre la población antigua y contemporánea (pp. 303-319). México: Instituto de Investigaciones Antropológicas, Universidad Nacional Autónoma de México.

Pinchi, V., De Luca, F., Ricciardi, F., Focardi, M., Piredda, V., Mazzeo, E. y Norelli, G. A. (2014). Skeletal age estimation for forensic purposes: A comparison of GP, TW2 and TW3 methods on an Italian sample. Forensic Science International, 238, 83-90. doi:https://doi. org/10.1016/j.forsciint.2014.02.030

Ramos Rodríguez, R. M. (2004). Homeorresis en la etapa formativa de la vida: estudio bioantropológico en menores de Tlaxiaco, Oaxaca (Tesis doctoral). Recuperado de http://132.248.9.195/ppt2004/0335291/Index.html

Ramos Rodríguez, R. M. (2005). Perfil morfológico de menores residentes en Tlaxiaco, Oaxaca: una propues- ta metodológica. Estudios de Antropología Biológica, 12(1), 451-471. http://revistas.unam.mx/index.php/eab/ article/view/19078

Sáenz Faulhaber, M. E. (1980). Crecimiento y maduración diferencial en una zona marginada de la capital mexicana. Anales de Antropología, 17(1): 281-294. doi: http:// dx.doi.org/10.22201/iia.24486221e.1980.1

Sáenz Faulhaber, M. E. (1981). Maduración ósea en una población rural de México. Anales de Antropología, 18(1), 271-285. http://revistas.unam.mx/index.php/antropologia/article/view/23949

Sáenz Faulhaber, M. E. (1989). Edad ósea y menarquia durante la adolescencia. Anales de Antropología, 26, 465474. http://revistas.unam.mx/index.php/antropologia/ article/view/13073

Schmeling, A., Reisinger, W., Geserick, G. y Olze, A. (2005). The current state of forensic age estimation of live subjects for the purpose of criminal prosecution. Forensic Science, Medicine and Pathology, 1(4), 239246. doi:https://doi.org/10.1385/FSMP:1:4:239

Schmeling, A., Reisinger, W., Loreck, D., Vendura, K., Markus, W. y Geserick, G. (2000). Effects of ethnicity on skeletal maturation: consequences for forensic age estimations. International Journal of Legal Medicine, 113(5), 253-258. doi:https://doi.org/10.1007/ s0041499001

Schmidt, S., Nitz, I., Schulz, R. y Schmeling, A. (2008). Applicability of the skeletal age determination method of Tanner and Whitehouse for forensic age diagnostics. International Journal of Legal Medicine, 122(4), 309314.

Tanner, J. M. (1978). Fetus into man. Physical growth from conception to maturity. Cambridge, Massachusetts, Harvard University Press.

Tanner, J. M., Whitehouse, R. y Healy, M. (1962). A new system for estimating skeletal maturity from the hand and wrist, with standards derives from a study of 2,600 healthy British children. Part II: The scoring system. París, Francia: Centre International de L'Enfance.

Tanner, J. M., Whitwhouse, R. H., Cameron, N., Marshall, W. A., Healy, M. J. R. y Goldstein, H. (2001). Assessment of skeletal maturity and prediction of adult height (TW3 method). London. W.B. Saunders LTD.

Tanner, J. M., Whitehouse, R. H., Marshall, W. A., Healy, M. J. y Goldstein, H. (1975). Assessment of skeletal maturity and prediction of adult height (TW2 method). Londres, Reino Unido: Academic Press.

Todd, T. W. (1937). Atlas of skeletal maturation. Part I, The hand. St. Louis: CV Mosby.

Villanueva, M, Sáenz, ME y Serrano, C (1984). Crecimiento y desarrollo en escolares de la Villa de Las Margaritas, Chiapas, México. Estudios de Antropología Biológica, 2, 427-452. http://revistas.unam.mx/index.php/eab/article/view/34675

Ye, Y. Y., Wang, C. X. y Cao, L. Z. (1992). Skeletal maturity of the hand and wrist in Chinese children in Changsha assessed by TW2 method. Annals of Human Biology, 19(4), 427-430. doi:https://doi. org/10.1080/03014469200002282 